\title{
Recent Progress of Fiber Loop Mirror-Based Sensors in China Jiliang University
}

\author{
Chunliu ZHAO, Yongxing JIN, Juan KANG, Huaping GONG, and Xinyong DONG
}

Institute of Optoelectronic Technology, China Jiliang University, Hangzhou, 310018, China

*Corresponding author: Chunliu ZHAO_E-mail: zhchunliu@hotmail.com

\begin{abstract}
The authors overview recent progress of fiber loop mirror based sensors in China Jiliang University. With the unique characteristics of fiber loop mirror made by various inserted fibers, fiber loop mirror based sensors have the potential to be exploited in a variety of wide applications.
\end{abstract}

Keywords: Optical fiber sensor, fiber loop mirror, photonic crystal fiber, pressure-induced birefringent fiber

\section{Introduction}

Fiber loop mirror (FLM), also called Sagnac interferometer, is interesting and very useful component used in optical devices and systems [1-6]. In FLM, two interfering waves counter-propagate through the same fiber and are exposed to the same environment. This makes it less sensitive to noise from the environment. An FLM can be made by inserting a highly birefringent (HiBi) fiber, a pressure-induced birefringent single-mode fiber, or a lowly birefringent fiber. Various kinds of FLM-based sensors have been demonstrated [7-23]. In this paper, a brief overview of the recent progress of FLM-based sensors in China Jiliang University is presented.

\section{Sensing principle}

The principle of operation of the FLM has been described previously [1, 2], therefore we will summarize the essentials here. As shown in Fig. 1, a 3-dB coupler splits the input signal equally into two counter-propagating waves which subsequently recombine (at the coupler) after propagating around the loop. The interference of the counter-propagating waves will be constructive or destructive, depending on the birefringence of the cavity, and thus, the loop transmission response is wavelength dependent. The phase difference between the fast and slow beams that propagate in the inserted fiber is given by

$$
\theta=2 \pi B L / \lambda
$$

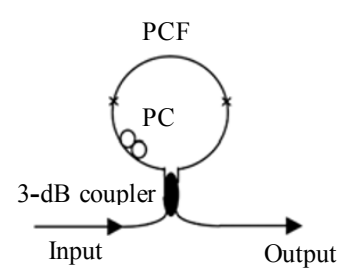

Fig. 1 Experimental setup of the FLM made of a PCF.

where $B, L$, and $\lambda$ are, respectively, the birefringence of the inserted fiber, the length of the inserted fiber, and the wavelength. When the variation of $B$ following the wavelength is small, there is $B=\left|n_{x}-n_{y}\right|$, where $n_{x}$ and $n_{y}$ are the effective refractive indices for each polarization mode. Ignoring insertion loss of the $3-\mathrm{dB}$ coupler and the fiber attenuation in the loop, the transmission spectrum of the fiber loop is approximately a periodic function of the wavelength, namely

$$
T=(1-\cos \theta) / 2 \text {. }
$$

The transmission dip wavelength is the resonant

Received: 19 August 2011 / Revised version: 18 September 2011

(C) The Author(s) 2011. This article is published with open access at Springerlink.com 
wavelength satisfying $2 \pi B L / \lambda_{\text {dip }}=2 k \pi$, where $k$ is any integer. Thus, the resonant dip wavelength can be described as

$$
\lambda_{\text {dip }}=B L / k \text {. }
$$

And the wavelength spacing between transmission dips can be expressed as

$$
S=\lambda^{2} / B L \text {. }
$$

When some variations (strain or temperature) are applied on the sensing fiber, they will cause the birefringence change $\Delta B$ and length change $\Delta L$. So the $\lambda_{\text {dip }}$ has a change which can be expressed as $[7,8]$

$$
\Delta \lambda_{\text {dip }}=(L \Delta B+B \Delta L) / k \text {. }
$$

So the change of varies can be obtained by measuring the wavelength shift of the dip in the output spectrum.

\section{Sensors based on FLMs made of highly-birefringent fibers}

When an FLM is inserted with a HiBi fiber, such as a polarization-maintaining fiber (PMF) (bow-tie, elliptical core, or Panda), a HiBi-FLM is made. In general, HiBi-FLM has several advantages, including input polarization independence and high extinction ratio, in addition to the good environmental stability and low cost.

Various kinds of sensors based on HiBi-FLMs have been proposed and realized since HiBi-FLMs are sensitive to many parameters and have high sensitivity, such as temperature sensors, level liquid sensors, refractive index sensors, strain sensors, and biochemical sensors [7-15]. In our recent work, a HiBi-FLM also showed a good sensitivity to curvature [10]. The sensitivity of the curvature measurement of $0.0344 \mathrm{~m}^{-1} \cdot \mathrm{pm}^{-1}$ was experimentally achieved in the range of $9.3 \mathrm{~m}^{-1}-21.7 \mathrm{~m}^{-1}$.

\subsection{Simultaneous measurement of temperature and other parameters in a HiBi-FLM sensor}

One serious problem of sensors based on a HiBi-FLM used in measurements of curvature, strain or other parameters is their cross-sensitivity to temperature, which may degrade sensors' performance since the optical path length of the HiBi-FLM shows temperature dependence caused by thermal refractive-index change and thermal expansion effect. Different methods have been demonstrated to discriminate or eliminate the temperature effect [11-13].

One of our suggestions is based on a HiBi-FLM with a long-period grating (LPG) inscribed in the HiBi-fiber [13], as shown in Fig. 2. Figure 3 shows

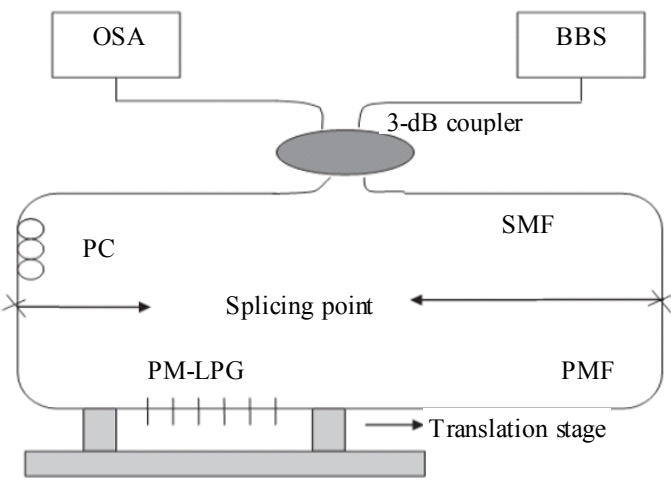

Fig. 2 Schematic of the proposed HiBi-FLM sensor with a inscribed HiBi-LPG.

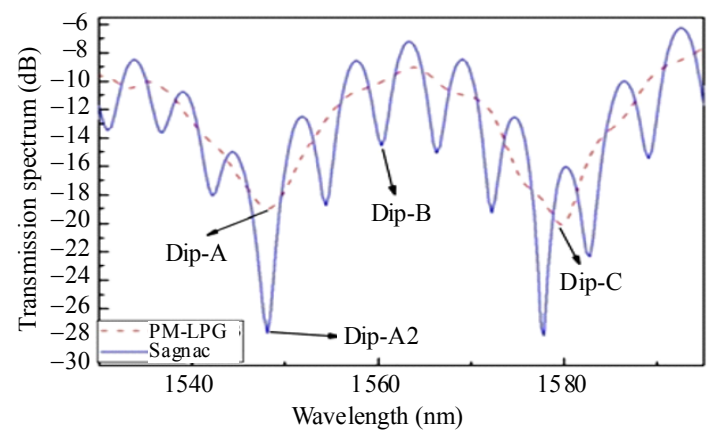

Fig. 3 Transmission spectra of the PM-LPG (dash line) and the HiBi-FLM (solid line).

the transmission spectrum of the HiBi-FLM, which is modulated by the dash line from the transmission spectrum of PM-LPG. The LPG inscribed in $\mathrm{HiBi}$-fiber was fabricated by a $\mathrm{CO}_{2}$ laser, and two resonance wavelengths were observed due to the birefringence of the HiBi-fiber. When one of the resonance dips in transmission spectrum was monitored, its intensity changed with applied strain and its wavelength varied with temperature change. 
By using a matrix equation, simultaneous strain and temperature measurements were realized.

\subsection{Temperature independent sensors based on a HiBi-FLM made of a highly birefringent photonic crystal fiber}

Photonic crystal fiber (PCF) incorporates a number of air holes that run along the length of the fiber [16-18]. By arranging the geometry or distribution of the core and the air-hole cladding, PCFs can be ultra-high birefringent, therefore HiBi-PCFs have been the best choice to make FLMs $[6,7,14,15]$. At the same time, unlike conventional PMFs, which contain at least two different glasses, each with a different thermal expansion coefficient, thereby causing the polarization of the propagation wave to vary with changing temperature, the PCF birefringence is highly insensitive to temperature because it is made of only one material (and air holes). Thus, the measurement is inherently temperature insensitive due to the great thermal stability of HiBi-PCF based FLMs. In our proposed strain sensors based on HiBi-PCF FLMs [7, 19], the length of $\mathrm{HiBi}-\mathrm{PCF}$ is shortened to several centimeters, and the temperature sensitivity is only about $0.3 \mathrm{pm} /{ }^{\circ} \mathrm{C}$, which is about 3000 times lower than the reported value of $0.94 \mathrm{~nm} /{ }^{\circ} \mathrm{C}$ of the HiBi-FLM temperature sensor [8].

\subsection{Highly sensitive temperature sensor based on an FLM made of an alcohol-filled HiBi-PCF}

HiBi-PCFs have a low thermo-optic and thermo-expansion coefficient, so HiBi-PCF FLMs can not be used to measure temperature directly. Recently, we proposed a novel FLM temperature sensor [20]. By inserting a short alcohol-filled HiBi-PCF into an FLM, a temperature sensor with an extremely high sensitivity can be realized by measuring the wavelength shift of the resonant dips of the alcohol-filled HiBi-PCF FLM.

For the FLM made of the alcohol-filled PCF, the resonant dips of the output spectrum are quite sensitive to the change of the refractive index (RI) of the filled alcohol. Figure 4 shows the temperature dependence of the refractive index of alcohol and the theoretical temperature dependence of the birefringence of the alcohol-filled PCF. With the temperature rising, the refractive index of alcohol decreases linearly, while the birefringence of the alcohol-filled PCF increases linearly.

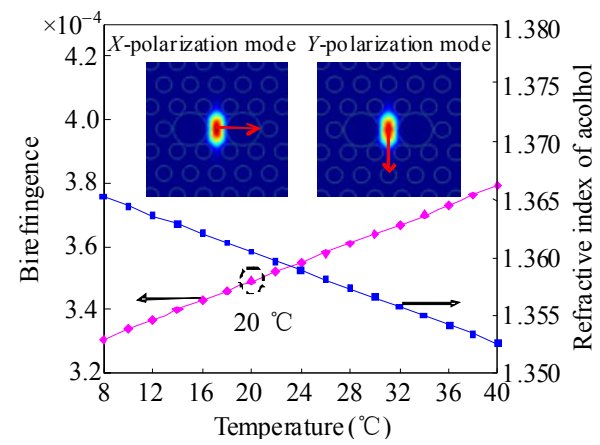

Fig. 4 Temperature dependence of the refractive index of alcohol and the birefringence of the alcohol-filled PCF in theory.
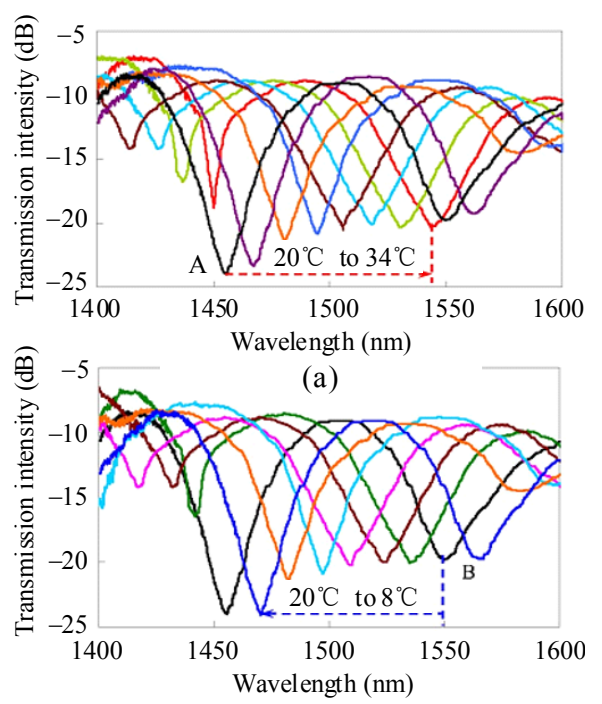

(b)

Fig. 5 Transmission spectra of the alcohol-filled HiBi-PCF FLM at different temperatures: (a) $20{ }^{\circ} \mathrm{C}$ to $34^{\circ} \mathrm{C}$ and (b) $8{ }^{\circ} \mathrm{C}$ and $20^{\circ} \mathrm{C}$.

Figures 5(a) and 5(b) show the transmission spectra of the alcohol-filled HiBi-PCF FLM at temperature ranges of $20{ }^{\circ} \mathrm{C}$ to $34{ }^{\circ} \mathrm{C}$ and $8{ }^{\circ} \mathrm{C}$ to $20{ }^{\circ} \mathrm{C}$, respectively. Dip A red-shifts about $88 \mathrm{~nm}$ with temperature increasing gradually from $20{ }^{\circ} \mathrm{C}$ to $34{ }^{\circ} \mathrm{C}$, while dip B blue-shifts about $80 \mathrm{~nm}$ with the temperature decreasing gradually from $20{ }^{\circ} \mathrm{C}$ to $8{ }^{\circ} \mathrm{C}$. 
The relationships between temperature and the resonant wavelength of dip A and dip B are linearity with the fitting degrees as high as 0.9997 and 0.9995 , respectively. The experimental temperature sensitivities of dip A and dip B are about $6.2 \mathrm{~nm} /{ }^{\circ} \mathrm{C}$ and about $6.6 \mathrm{~nm} /{ }^{\circ} \mathrm{C}$, respectively. The temperature sensitivity of the alcohol-filled PCF FLM is very high, and reach up to about 660 and 7 times higher than that of an FBG $\left(\sim 0.01 \mathrm{~nm} /{ }^{\circ} \mathrm{C}\right)$ and that of the FLM made of a conventional high birefringent fiber with a $72-\mathrm{cm}$ length [8].

\section{Sensors based on an FLM made of a pressure-induced birefringent single-mode fiber}

FLM sensors mentioned above were all based on a HiBi fiber (PMF, HiBi-PCF, or filled-HiBi-PCF). When two ends of the HiBi fiber were connected to two ports of a 3-dB coupler by using a normal fusion splicer, there was more than $6-\mathrm{dB}$ insertion loss due to the mismatching of mode field and numerical aperture between the single-mode fiber (SMF) and the HiBi fiber [6, 7].

In recent years, the pressure-induced birefringence in an SMF has been theoretically analyzed, and pressure-induced birefringence for pressure vector sensing based on SMF has been reported. Our group firstly demonstrated a temperature sensor based on an FLM made of a pressure-induced birefringent SMF [21].

The proposed FLM temperature sensor is shown in Fig. 6. The pressure-induced birefringent SMF was formed by pressing the SMF with the core and cladding which was placed between two 5-cm-length flat plates. The physical sensing mechanism is an increase of the fiber birefringence in the FLM with an increase of temperature, and the resonant wavelength of the loop shifts to larger values as temperature increases. Figure 7(a) shows several measured transmission spectra around the transmission minimum at $1558.6 \mathrm{~nm}$ under different temperatures. The measured data are shown in
Fig. 7 (b). A linear fitting to the experimental data gives a wavelength-temperature sensitivity of $0.65 \mathrm{~nm} /{ }^{\circ} \mathrm{C}$, which shows that the linearity of the wavelength to temperature response is excellent.

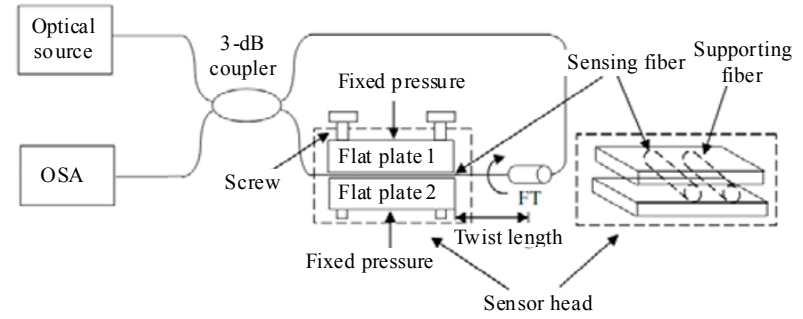

Fig. 6 Schematic diagram of the proposed FLM temperature sensor (FT: fiber twister).

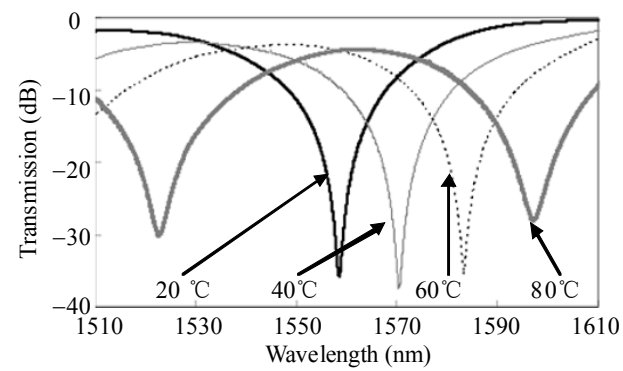

(a)

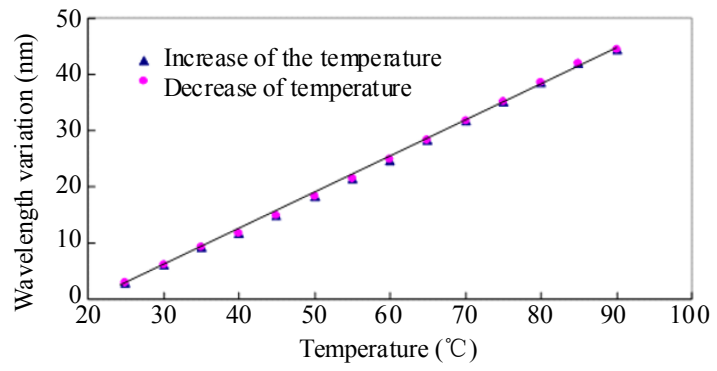

(b)

Fig. 7 Properties of the proposed FLM with temperature: (a) measured transmission spectra at different temperatures and (b) wavelength variation of the transmission dip at $1558.6 \mathrm{~nm}$ against temperature.

In another application, a temperature-insensitive transverse mechanical load sensor was realized by using a pressure-induced biregringent PCF-based FLM [22]. Figure 8 shows the configuration of the proposed sensor. A metal plate was used to introduce initial birefringence by applying a lateral force on a section of a solid core PCF. The birefringence induced by the initial transverse force can be adjusted so that the output period and the 
transmission minima of the fringes can be tuned without need to cut the fiber length with great accuracy. As shown in Fig. 9, the dip wavelength shifts toward the red side when the load increases from $0 \mathrm{~g}$ to $889.45 \mathrm{~g}$. The linearity of the relationship between the dip wavelength and the applied load is very good, and the sensitivity of the sensor is about $0.519 \mathrm{~nm} \cdot \mathrm{N}^{-1}$.

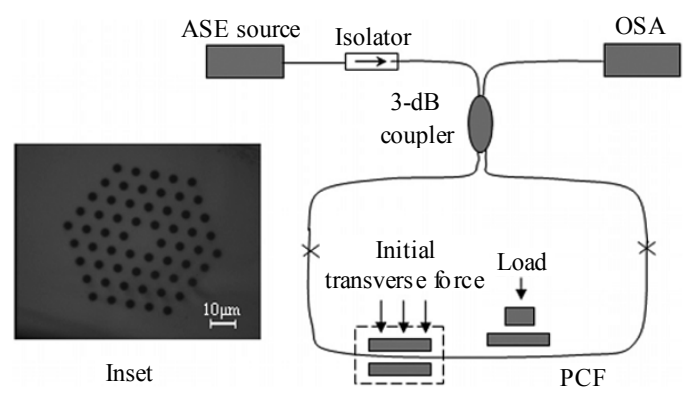

Fig. 8 Schematic diagram of the experimental setup.

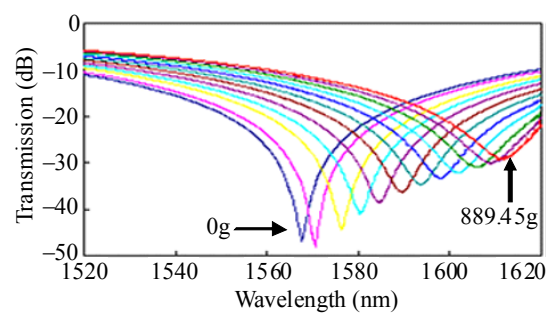

Fig. 9 Measured transmission spectra under different mechanical loads.

\section{Sensors based on an FLM made of a lowly birefringent fiber with large measurement range}

A lowly birefringent fiber also can be used to form an FLM. The advantage of FLM sensors based on lowly birefringent fiber is larger measurement range compared with that based on a HiBi fiber or a pressure-induced birefringent SMF, which is important in some applications.

In [23], we demonstrated an optical fiber strain sensor based on a lowly birefringent PCF FLM. The FLM was formed by a PCF with the birefringence value of $5.8 \times 10^{-5}$. Due to the low birefringence, just one dip in the wavelength range of $1500 \mathrm{~nm}-$ $1600 \mathrm{~nm}$ appeared, while the HiBi-fiber based Sagnac sensor had a small fringe separation, which could lead to the overlap of the fringes when the wavelength shift was larger than the fringe separation [6, 7]. Thus the measurement range increased largely. The sensitivity of the strain measurement of $0.457 \mathrm{pm} / \mu \varepsilon$ was achieved. The same experimental setup also could be used as an optical fiber curvature sensor [24]. The sensitivity of the curvature measurement of $0.337 \mathrm{~nm} / \mathrm{m}^{-1}$ was achieved in the range of $0-9.92 \mathrm{~m}^{-1}$.

\section{Demodulation of FLM sensors}

In general, FLM sensors are based on monitoring the resonant wavelength variation of the FLM. In these configurations, a broadband light source and an optical spectrum analyzer (OSA) are needed, which cause the sensors expensive. A stable demodulation system for an FLM based sensor is important for practical applications.

\subsection{Intensity measurement for a HiBi-PCF FLM strain sensor by using a single-wavelength light}

Based on the fact that the transmission intensity of an FLM at a fixed wavelength is strongly affected by the strain applied on a piece of HiBi-PCF in the FLM since the transmission spectrum of the FLM shifts with the applied strain, but the resonant dip (both wavelength and intensity) is insensitive to temperature, we [19] proposed a low-cost temperature-insensitive strain sensor based on a HiBi-PCF FLM, in which a distributed-feedback (DFB) laser was used as the light source. Since the output intensity of the FLM is directly proportional to the applied strain, only an optical power meter is sufficient to detect strain variation without the need for an expensive OSA. When the applied strain is $3000 \mu \varepsilon$, the strain sensitivity is about $2.7 \mathrm{~dB} / 1000 \mu \varepsilon$ at the fixed wavelength $1530 \mathrm{~nm}$. A strain resolution of $0.37 \mu \varepsilon$ is achieved when an optical power meter with an intensity resolution of $0.001 \mathrm{~dB}$ is used, which is 24.6 times better than that of the strain sensor based on the resonant wavelength measurement [7]. 


\subsection{Demodulation based on a long-period grating in PCF and a band-pass filter for a temperature FLM sensor}

For the principle of the proposed demodulation in [25], a long-period grating in PCF (PCF-LPG) serves as a temperature insensitive filter to provide wavelength dependent optical power transmission and a band-pass filter with a broadband light source is to provide a narrow band light source. By utilizing the stable filtering function of the PCF-LPG, the resonant wavelength variation of the FLM with temperature is transferred effectively to the intensity variation of the output light. When the optical intensity of the output is monitored, temperature applied on the FLM will be deduced.

Figure 10 shows the transmission spectra of the FLM, the PCF-LPG, and the band-pass filter. When temperature increases, the transmission spectrum of the FLM blue-shifts due to thermally induced refractive index change and thermal expansion of the panda fiber, but the transmission spectra of the LPG in PCF are stable. Due to the filtering function of the LPG in PCF, the intensity of the resonant peak in gray region increases while the resonant peaks shift to shorter wavelength with an increase of temperature. Therefore, when a band-pass filter is used, as shown in Fig. 10, the part of the output light outside the band of the filter is attenuated owing to the function of the band-pass filter. And the intensity of the remainder will change with the transmission peak of the HiBi-FLM shifting. Therefore, converting the wavelength variation of the HiBi-FLM into intensity variation in the output of the LPG directly is realized.

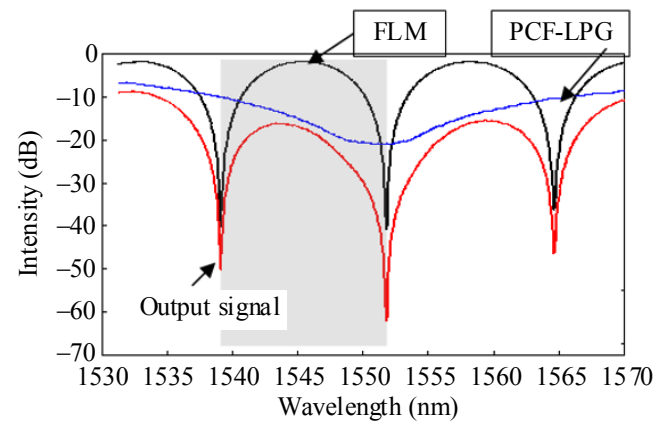

Fig. 10 Transmission spectra of the FLM, the LPG in PCF and the output signal.

Figure 11 shows the relationships of the output intensity and temperature when different filters (with the same center wavelength at $1545 \mathrm{~nm}$, but different bandwidths) are used. Table 1 shows the properties of the proposed FLM temperature sensor when different filters are used. When the filter is chosen a bandwidth of $3 \mathrm{~nm}$ with a center wavelength at $1545 \mathrm{~nm}$, the average sensitivity of the proposed FLM temperature sensor is about $1.742 \mathrm{~dB} /{ }^{\circ} \mathrm{C}$. When a power meter with the resolution of $0.01 \mathrm{~dB} \cdot \mathrm{m}$ is used, the temperature resolution is obtained to be $0.006{ }^{\circ} \mathrm{C}$.

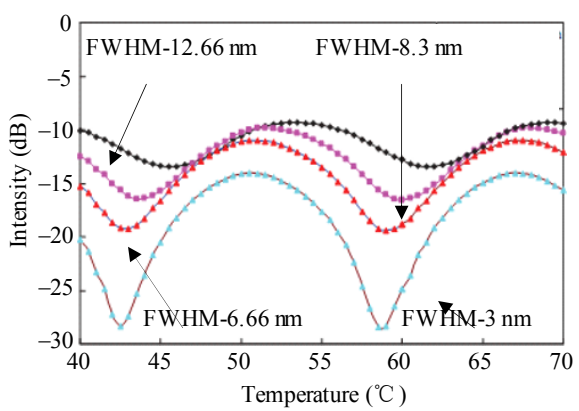

Fig. 11 Relationships of the output power and temperature when different filters are used.

Table 1 Properties of the proposed FLM temperature sensor when different filters are used.

\begin{tabular}{l|l|l|l|l}
\hline \multicolumn{1}{c|}{ PWHM } & \multicolumn{1}{|c|}{$12.66 \mathrm{~nm}$} & \multicolumn{1}{c|}{$8.3 \mathrm{~nm}$} & \multicolumn{1}{c|}{$6.66 \mathrm{~nm}$} & \multicolumn{1}{c}{$3 \mathrm{~nm}$} \\
\hline Properties monotone interval & $45.5{ }^{\circ} \mathrm{C} \sim 53.5{ }^{\circ} \mathrm{C}$ & $43.5{ }^{\circ} \mathrm{C} \sim 51.5{ }^{\circ} \mathrm{C}$ & $43{ }^{\circ} \mathrm{C} \sim 51{ }^{\circ} \mathrm{C}$ & $42.5{ }^{\circ} \mathrm{C} \sim 50.5{ }^{\circ} \mathrm{C}$ \\
\hline Output intensity range & $-13.4664 \mathrm{~dB} \sim-9.3291 \mathrm{~dB}$ & $-16.4375 \mathrm{~dB} \sim-9.8497 \mathrm{~dB}$ & $-19.2807 \mathrm{~dB} \sim-10.9793 \mathrm{~dB}$ & $-28.3572 \mathrm{~dB} \sim-14.0612 \mathrm{~dB}$ \\
\hline \multirow{2}{*}{ Fitting function } & $y=-0.0227 x^{3}+3.3477 x^{2}-$ & $y=-0.0249 x^{3}+3.5056 x^{2}-$ & $y=-0.0188 x^{3}+2.5377 x^{2}-$ & $y=0.0068 x^{3}-1.2038 x^{2}+$ \\
& $163.52 x+2636.6$ & $163.51 x+2509.1$ & $112.84 x+1632.7$ & $69.702 x-1337.4$ \\
\hline$R^{2}$ & 0.9978 & 0.9988 & 0.9987 & 0.9979 \\
\hline Average sensitivity & $0.783 \mathrm{~dB} /{ }^{\circ} \mathrm{C}$ & $1.082 \mathrm{~dB} /{ }^{\circ} \mathrm{C}$ & $1.222 \mathrm{~dB} /{ }^{\circ} \mathrm{C}$ & $1.742 \mathrm{~dB} /{ }^{\circ} \mathrm{C}$ \\
\hline
\end{tabular}




\subsection{Demodulation based on a PCF-LPG with differential processing for an FLM temperature sensor}

In [26], the proposed demodulation system is constructed with a PCF-LPG, a 3-dB coupler, and two band-pass filters with the full width at half maximum (FWHM) $6.66 \mathrm{~nm}$ whose center wavelengths are $1542 \mathrm{~nm}$ and $1561.77 \mathrm{~nm}$. By utilizing the stable filtering function of the PCF-LPG, the variations of the FLM's resonant wavelengths with temperature, which are located within the positive and negative linear regions of the LPG's transmission spectrum respectively, are transferred effectively to the intensity variations simultaneously. The intensity signals provided by the PCF-LPG are separated effectively by the two band-pass filters. Both signals $W_{1}$ and $W_{2}$ are related with the measurand, while they also may include the all of fluctuation of the sensor system. When monitoring $W_{1}$ or $W_{2}$, we can obtain the information of temperature applied on the FLM sensor, but the accuracy will be low due to the effect of noises. $W_{1}$ and $W_{2}$ are gotten at the same condition since the light from the broadband source passes through the same path and input to the monitor. By use of the differential processing $\Delta W=\left(W_{1}-W_{2}\right) /\left(W_{1}+W_{2}\right)$, the measurement is free from the effect of power fluctuations of the light source and any other noises.

The relationship of the differential $\Delta W$ with temperature remains stable when the power of the light source changes $\pm 10 \%$, and the differential algorithm based on a PCF-LPG eliminates the noises effectively, as shown in Fig. 12. Compared with the

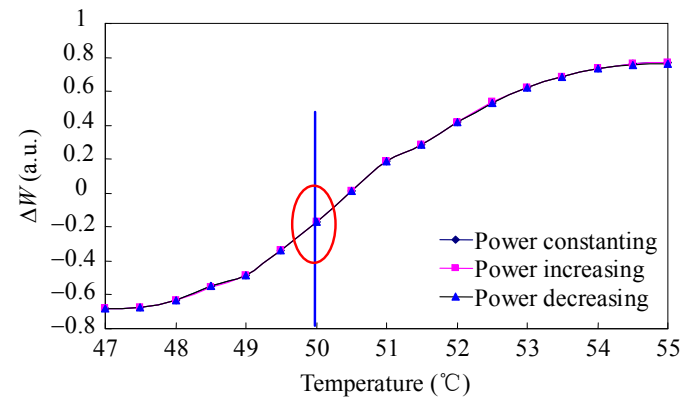

Fig. 12 Relationship of $\Delta W$ and temperature when the power of light source changes. result without the differential $\Delta W$, the accuracy of the HiBi-FLM sensor rises from $90.5 \%$ to $99.7 \%$.

\section{Conclusions}

In conclusion, we have reviewed our recent progress on FLM-based sensors. Different kinds of FLM are realized. With the unique characteristics of FLM made by various inserted fibers, it is expected that they would play an important role in the field of fiber optic sensors.

\section{Acknowledgement}

This work was supported by the National Basic Research Program of China (973 Program) under Grant No. 2010CB327804, the National Natural Science Foundation of China under Grant No. 61108058, the major project of Education Department in Zhejiang Province of China under Grant No. Z200909231 and the Qianjiang Talents Program of Zhejiang Province of China under Grant No. QJD0902005.

Open Access This article is distributed under the terms of the Creative Commons Attribution License which permits any use, distribution, and reproduction in any medium, provided the original author(s) and source are credited.

\section{References}

[1] V. Vali and R. W. Shorthill, "Fiber ring interferometer," Appl. Opt., vol. 15, no. 5, pp. 1099-1103, 1976.

[2] D. B. Mortimore, "Fiber loop reflectors," $J$. Lightwave Technol., vol. 6, no. 7, pp. 1217-1224, 1988.

[3] X. Fang and R. O. Claus, "Polarization-independent all-fiber wavelength-division multiplexer based on a Sagnac interferometer," Opt. Lett., vol. 20, no. 20, pp. 2146-2148, 1995.

[4] S. Li, K. S. Chiang, and W. A. Gambling, "Gain flattening of an erbium-doped fiber amplifier using a high-birefringence fiber loop mirror," IEEE Photon. Technol. Lett., vol. 13, no. 9, pp. 942-944, 2001.

[5] L. Yuan, W. Jin, L. Zhou Y. L. Hoo, and M. S. Demokan, "Enhancement of multiplexing capability of low-coherence interferometric fiber sensor array by 
use of a loop topology," J. Lightwave Technol., vol. 21, no. 5, pp. 1313-1319, 2003.

[6] C. L. Zhao, X. Yang, C. Lu, W. Jin, and M. S. Demokan, "Temperature-insensitive interferometer using a highly berefringent photonic crystal fiber loop mirror," IEEE Photon. Technol. Lett., vol. 16, no. 11, pp. 2535-2537, 2004.

[7] X. Dong, H. Y. Tam, and P. Shum, "Temperature-insensitive strain sensor with polarization-maintaining photonic crystal fiber based Sagnac interferometer," Appl. Physics Lett., vol. 90, no. 15 , pp. $151113,2007$.

[8] Y. Liu, B. Liu, X. Feng, W. Zhang, G. Zhou, S. Yuan, G. Kai, and X. Dong, "High-birefringence fiber loop mirrors and their applications as sensors," Appl. Opt., vol. 44, no. 12, pp. 2382-2390, 2005.

[9] O. Frazão, B. V. Marquesa, P. Jorge, J. M. Baptista, and J. L. Santos, "High birefringence D-type fibre loop mirror used as refractometer," Sensors and Actuators B: Chemical, vol. 135, no. 1, pp. 108-111, 2008.

[10] Y. Zhao, Y. Jin, H. Liang, J. Wang, and X. Dong, "Curvature sensor based on fiber loop mirror with polarization maintaining fiber," Microwave and Optical Technol. Lett., vol. 53, no. 9, pp. 2066-2068, 2011.

[11] Y. G. Han, Y. Chung, and S. B. Lee, "Discrimination of strain and temperature based on a polarization-maintaining photonic crystal fiber incorporating an erbium-doped fiber," Opt. Commun., vol. 282, no. 11, pp. 2161-2164, 2009.

[12] C. L. Zhao, J. Zhao, W. Jin, J. Ju, L. Cheng, and X. Huang, "Simultaneous strain and temperature measurement using a highly birefringence fiber loop mirror and a long period grating written in a photonic crystal fiber," Opt. Commun., vol. 282, no. 20, pp. 4077-4080, 2009.

[13] J. Kang, X. Dong, C. L. Zhao, W. Qian, and M. Li, "Simultaneous measurement of strain and temperature with a long-period fiber grating inscribed Sagnac interferometer," Opt. Commun., vol. 284, no. 8, pp. 2145-2148, 2011.

[14] X. Yang, C. L. Zhao, Q. Peng, X. Zhou, and C. Lu, "FBG sensor interrogation with high temperature insensitivity by using a HiBi-PCF Sagnac loop filter," Opt. Commun., vol. 250, no. 1-3, pp. 63-68, 2005.

[15] H. Y. Fu, H. Y. Tam, L. Y. Shao, X. Dong, P. K. A. Wai, C. Lu, and Sunil K. Khijwania, "Presure sensor realized with polarization-maintaining photonic crystal fiber-based Sagnac interferometer," Appl. Opt., vol. 47, no. 15, pp. 2835-2839, 2008.

[16] T. P. Hansen, J. Broeng, Stig E. B. Libori, E. Knudsen, A. Bjarklev, J. R. Jensen, and H. Simonsen,
"Highly birefringent index-guiding photonic crystal fibers," IEEE Photon. Technol. Lett., vol. 13, no. 6, pp. 588-590, 2001.

[17] A. Ortigosa-Blanch, J. C. Knight, W. J. Wadsworth, J. arriaga, B. J. Mangan, T. A. Birks, and P. St. J. Russell, "Highly birefringent photonic crystal fibers," Opt. Lett., vol. 25, no. 18, pp. 1325-1327, 2000.

[18] K. Suzuki, H. Kubota, S. Kawanishi, H. Kubota, and S. Kawanishi, "Optical properties of a low-loss polarization-maintaining photonic crystal fiber," Optics Express, vol. 9, no. 13, pp. 676-680, 2001.

[19] W. Qian, C. L. Zhao, X. Dong, and W. Jin, "Intensity measurement based temperature independent strain sensor using a highly birefringent photonic crystal fiber loop mirror," Opt. Commun., vol. 283, no. 24, pp. 5250-5254, 2010.

[20] W. Qian, C. L. Zhao, S. He, X. Dong, S. Zhang, Z. Zhang, S. Jin, J. Guo, and H. Wei, "High-sensitivity temperature sensor based on an alcohol-filled photonic crystal fiber loop mirror," Opt. Lett., vol. 36, no. 9, pp. 1548-1550, 2011.

[21] Y. Jin, C. C. Chan, Y. Zhang, X. Dong, and P. Zu, "Temperature sensor based on a pressure-induced birefringent single-mode fiber loop mirror," Meas. Sci. Technol., vol. 21, no. 6, pp. 065204, 2010.

[22] P. Zu, C. C. Chan, Y. Jin, Y. Zhang, and X. Dong, "Fabrication of a temperature-insensitive transverse mechanical load sensor by using a photonic crystal fiber-based Sagnac loop," Meas. Sci. Technol., vol. 22, no. 2, pp. 025204, 2011.

[23] H. Gong, C. C. Chan, L. Chen, and X. Dong, "Strain sensor realized by using low-birefringence photonic-crystal-Fiber-based Sagnac loop," IEEE Photon. Technol. Lett., vol. 22, no. 6, pp. 1238-1240, 2010.

[24] H. Gong, C. C. Chan, P. Zu, L. Chen, and X. Dong, "Curvature measurement by using low-birefringence photonic crystal fiber based Sagnac loop," Opt. Commun., vol. 283, no. 16, pp. 3142-3144, 2010.

[25] Y. Wang, C. L. Zhao, X. Dong, J. Kang, and S. Jin, "A fiber loop mirror temperature sensor demodulation technique using a long-period grating in a photonic crystal fiber and a band-pass filter," Review of Scientific Instruments, vol. 82, no. 7, pp. 073101, 2011.

[26] Y. Wang, C. L. Zhao, X. Dong, J. Kang, and S. Jin, "Demodulation based on a long-period grating in photonic crystal fiber with differential processing for high-birefringence fiber loop mirror temperature sensor," in Proc. SPIE(International Conference on Optical Fiber Sensors (OFS)), Ottawa, Canada, May 15-19, vol. 7753, 2011. 